This item was submitted to Loughborough's Research Repository by the author.

Items in Figshare are protected by copyright, with all rights reserved, unless otherwise indicated.

\title{
Space-time block coding for four transmit antennas with closed loop feedback over frequency selective fading channels
}

PLEASE CITE THE PUBLISHED VERSION

PUBLISHER

(c) IEEE

VERSION

VoR (Version of Record)

\section{LICENCE}

CC BY-NC-ND 4.0

\section{REPOSITORY RECORD}

Toker, Cenk, Sangarapillai Lambotharan, and Jonathon Chambers. 2019. "Space-time Block Coding for Four Transmit Antennas with Closed Loop Feedback over Frequency Selective Fading Channels”. figshare. https://hdl.handle.net/2134/5765. 
This item was submitted to Loughborough's Institutional Repository (https://dspace.lboro.ac.uk/) by the author and is made available under the following Creative Commons Licence conditions.

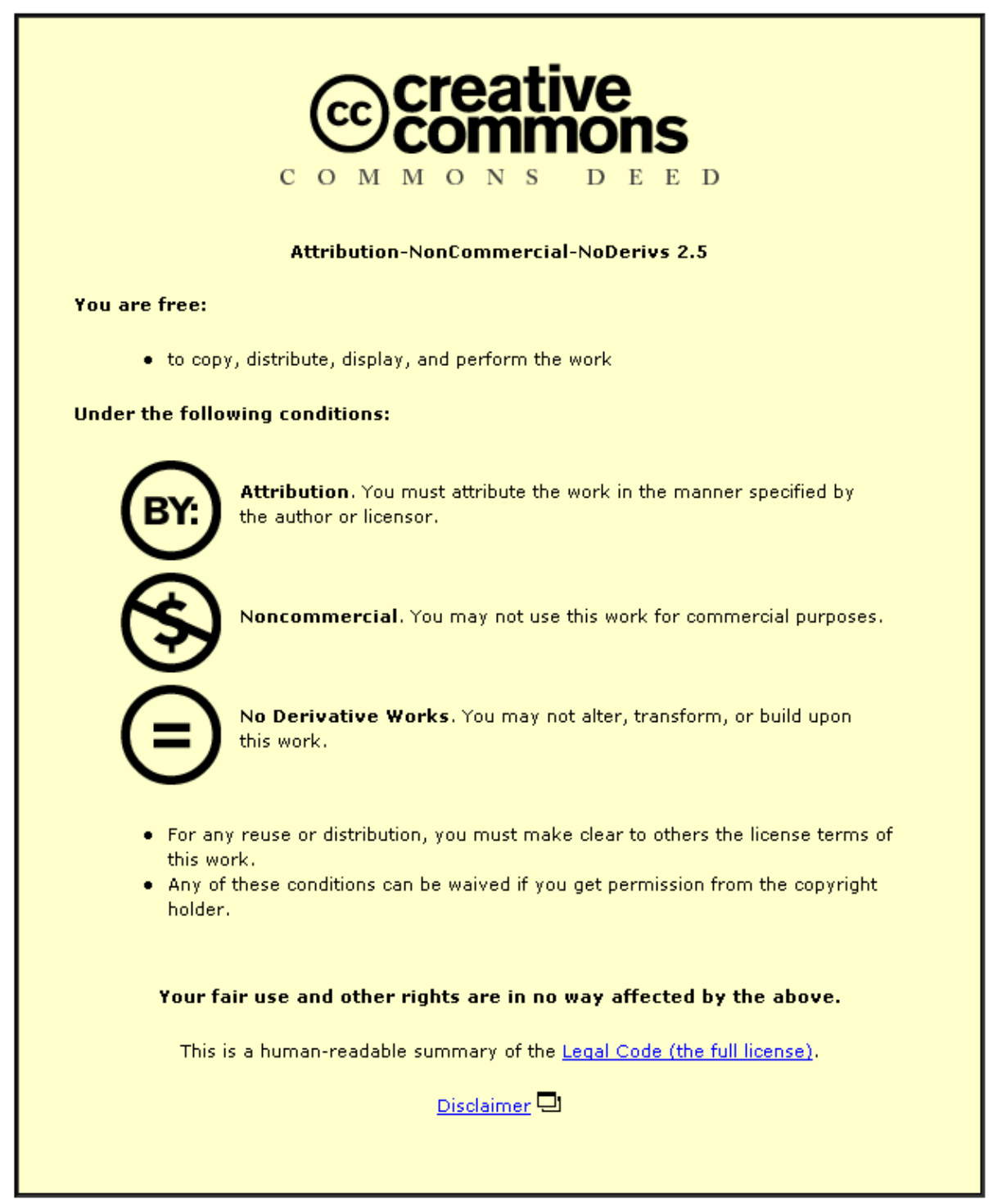

For the full text of this licence, please go to: http://creativecommons.org/licenses/by-nc-nd/2.5/ 


\title{
Space-Time Block Coding for Four Transmit Antennas with Closed Loop Feedback over Frequency Selective Fading Channels
}

\author{
Cenk Toker, Sangarapillai Lambotharan and Jonathon A. Chambers \\ Centre for DSP Research, King's College London, Strand Campus, London, WC2R 2LS, UK \\ \{cenk.toker, s.lambotharan, jonathon.chambers\}@kcl.ac.uk
}

\begin{abstract}
Orthogonal space-time block coding is a transmit diversity method that has the potential to enhance the forward capacity. For a communication system with complex alphabet, full diversity and full code rate space-time codes are available only for two antennas, and for more than two antennas full diversity is achieved only when the code rate is lower than one. A quasi-orthogonal code could provide full code rate, but at the expense of loss in diversity, which results into degradation in performance. In this paper, we propose a closed loop feedback scheme for quasi-orthogonal codes which provides full diversity while achieving the full code rate. We, in particular, investigate the performance of this scheme, when the feedback information is quantised and when the fading of the channel is frequency-selective.
\end{abstract}

\section{INTRODUCTION}

Transmit diversity, such as a space-time block code (STBC), has the potential to increase the link performance of a communication system. In [2], Alamouti proposed a two transmit antenna STBC which exploits full diversity and provides full code rate. It has also been shown in [8] that full diversity and full code rate block codes do not exist for complex valued symbols and for more than two transmit antennas. However, quasi-orthogonal codes have been proposed in ([3], [5], [6] and [7]) that provides full code rate at the expense of loss in diversity.

In [10] and [7], as a remedy to this non-orthogonality problem, a closed loop feedback scheme has been proposed. Accordingly, the signals from various antennas are rotated before transmission in a prescribed way based on the information provided by the receiver. The work in [7] considered unlimited feedback information, however, the work in [10] assumed a more realistic environment where only a finite number of bits are allowed for the feedback, and the performance was analyzed against quantization of the feedback information. Moreover, the performance for schemes based upon rotating the antenna signal from just a single antenna and from dual antennas have also been studied to demonstrate only two phase feedback is adequate to attain full diversity.

In this paper, we extend this work to investigate the performance of closed loop feedback schemes for a frequency selective fading channel. We show that the amount of feedback required remains the same as what is required for a flat fading channel, and full diversity can be achieved only with two phase feedback. Moreover, a significant gain in diversity is realized even with only one phase rotation with two bits per slot, for the feedback.

\section{Problem Statement}

A general transmission scheme for a four transmit antenna based closed-loop STBC is depicted in Figure 1. The rows of the transmission matrix (2) is transmitted from the four antennas, with one row at a symbol interval. For the single path case, the channel coefficients $\mathbf{h}=\left[\begin{array}{llll}h_{1} & h_{2} & h_{3} & h_{4}\end{array}\right]^{T}$ are independent complex valued Gaussian random variables with zero mean and unit variance, hence the amplitude is Rayleigh distributed. For multipaths, $h_{k}^{l}$ is the fading coefficient for the $l$-th path corresponding to the transmit antenna $k$. Without loss of generality, we assume a normalization such that $\sum_{l=0}^{L-1}\left|h_{k}^{l}\right|^{2}=1$, where $L$ is the number of multipaths.

In [8], it has been shown that a full diversity and full code rate complex valued space-time block codes exist only for a dimension of two. An example is the well-known Alamouti's scheme [2], which is expressed by the following transmit matrix

$$
\mathbf{C}_{12}=\left[\begin{array}{cc}
x_{1} & x_{2} \\
-x_{2}^{*} & x_{1}^{*}
\end{array}\right]
$$

which corresponds to two transmit antennas transmitting a symbol block of two symbols in two symbol intervals. The $n$-th row of $\mathbf{C}_{12}(n=1,2)$ corresponds to the symbols transmitted from n-th antenna at two consecutive symbol intervals. The subscript ' 12 ' is included to denote that the matrix contains symbols $x_{1}$ and $x_{2}$.

In [3] and [9], this scheme is extended to four antennas, which can be viewed as a family of quasi-orthogonal codes. The example in [3] is

$$
\mathbf{C}_{14}=\left[\begin{array}{cc}
\mathbf{C}_{12} & \mathbf{C}_{34} \\
-\mathbf{C}_{34}^{*} & \mathbf{C}_{12}^{*}
\end{array}\right]=\left[\begin{array}{cccc}
x_{1} & x_{2} & x_{3} & x_{4} \\
-x_{2}^{*} & x_{1}^{*} & -x_{4}^{*} & x_{3}^{*} \\
-x_{3}^{*} & -x_{4}^{*} & x_{1}^{*} & x_{2}^{*} \\
x_{4} & -x_{3} & -x_{2} & x_{1}
\end{array}\right]
$$

which is an extension of Alamouti's scheme, where $\mathbf{C}_{34}$ is used to denote the $2 \times 2$ matrix which contains symbols $x_{3}$ and $x_{4}$. First, assuming a flat fading environment, and a single receive antenna, the received signal (after complex conjugation of alternate symbols) over four symbol intervals can be expressed as

$$
\begin{gathered}
{\left[\begin{array}{c}
r_{1} \\
r_{2}^{*} \\
r_{3}^{*} \\
r_{4}
\end{array}\right]=\left[\begin{array}{cccc}
h_{1} & h_{2} & h_{3} & h_{4} \\
h_{2}^{*} & -h_{1}^{*} & h_{2}^{*} & -h_{3}^{*} \\
h_{3}^{*} & h_{2}^{*} & -h_{1}^{*} & -h_{2}^{*} \\
h_{4} & -h_{3} & -h_{2} & h_{1}
\end{array}\right]\left[\begin{array}{l}
x_{1} \\
x_{2} \\
x_{3} \\
x_{4}
\end{array}\right]+\left[\begin{array}{l}
n_{1} \\
n_{2}^{*} \\
n_{3}^{*} \\
n_{4}
\end{array}\right]} \\
\mathbf{r}=\mathbf{H} \mathbf{x}+\mathbf{n}
\end{gathered}
$$

where $n_{i}, i=1,2,3,4$ are the zero-mean, circularly symmetric, complex valued Gaussian noise terms with variance $\sigma_{n}^{2}$. 


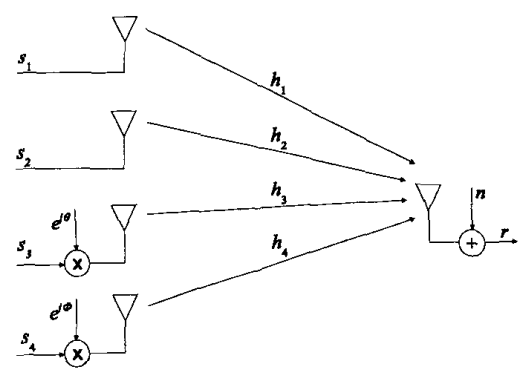

Figure 1: The new transmission scheme with 4 transmit and 1 receive antennas, showing the preprocessing phase terms, the fading channel coefficients and the additive white Gaussian noise

Applying channel matched filtering results in

$$
\mathbf{r}_{m f}=\mathbf{H}^{H} \mathbf{r}=\Delta \mathbf{x}+\tilde{\mathbf{n}}
$$

For an orthogonal block code, all the off-diagonal terms of $\boldsymbol{\Delta}=\mathbf{H}^{H} \mathbf{H}$ will be zero as in the Alamouti's scheme. But for quasi-orthogonal codes, some non-zero terms exist that reduce the diversity gain of the code. Our aim is to eliminate or reduce these off-diagonal elements. For the block code in equation (2),

$$
\boldsymbol{\Delta}=\left[\begin{array}{cccc}
\gamma & 0 & 0 & \alpha \\
0 & \gamma & -\alpha & 0 \\
0 & -\alpha & \gamma & 0 \\
\alpha & 0 & 0 & \gamma
\end{array}\right]
$$

where $\gamma=\sum_{k=1}^{4}\left|h_{k}\right|^{2}$, and $\alpha=\operatorname{Re}\left\{h_{1}^{*} h_{4}-h_{2}^{*} h_{3}\right\}$.Using various combination of symbols, several quasi-orthogonal codes can be generated, but all of them possess similar properties.

\section{ORTHOGONALIZATION BY FEEDBACK}

It can be shown that the non-zero off diagonal element in $\Delta$ reduces the diversity gain and the signal-to-noise ratio (SNR) at the receiver, hence our aim is to minimize this. In [10], we investigated this problem, independently from that of [7], for a four antenna scheme. We proposed to modify the signals transmitted from certain antennas by rotating with a proper phase angle such that the magnitude of the off-diagonal element $\alpha$ is minimized. Hence for the matrix in equation (4), we consider the following cost function

$$
|\alpha|=\left|\operatorname{Re}\left\{h_{1}^{*} h_{4}-h_{2}^{*} h_{3}\right\}\right| .
$$

If we multiply the first and second terms in the brackets in equation (5) by a phasor, it is possible to make $\alpha$ zero. Let us assume that we rotate the signal from the third and fourth antennas by $\theta$ and $\phi$ respectively. This is equivalent to multiplying the first term in the brackets in equation (5) by $e^{j \theta}$ and the second term by $e^{j \phi}$. Also defining $\kappa=h_{1}^{*} h_{4}$ and $\lambda=h_{2}^{*} h_{3}$, we could write

$$
\begin{aligned}
\alpha & =\operatorname{Re}\left\{h_{1}^{*} h_{4} e^{j \theta}-h_{2}^{*} h_{3} e^{j \phi}\right\} \\
& =|\kappa| \cos (\theta+\angle \kappa)-|\lambda| \cos (\phi+\angle \lambda)
\end{aligned}
$$

where $|\cdot|$ and $\angle$ denote the absolute value and the angle (arctan) operators, respectively.

After some manipulations, it can be shown that $\alpha=0$ has infinitely many solutions for $\theta$ and $\phi$. The solutions are

$$
\theta=\arccos \left(\frac{|\lambda|}{|\kappa|} \cos (\phi+\angle \lambda)\right)-\angle \kappa
$$

provided that

$$
\phi \in \begin{cases}{[0,2 \pi)} & \text {, when }|\lambda|<|\kappa| \\ {[\pi-\xi-\angle \lambda, \xi-\angle \lambda]} & \\ \cup[-\xi-\angle \lambda, \pi+\xi-\angle \lambda] & \text {,otherwise }\end{cases}
$$

where $\xi=\arccos \left(\frac{|\kappa|}{|\lambda|}\right)$.

\section{FREQUENCY-SELECTIVE CHANNEL}

As in the previous case, assume all the signal transmitted from every antenna is spread by the identical spreading sequence $s(t)$. Also assume that this spreading sequence has ideal autocorrelation property, i.e.

$$
\int_{0}^{T} s(t) s^{*}(t-\tau) d t= \begin{cases}1 & , \tau=0 \\ 0, & \tau \neq 0\end{cases}
$$

where $T$ is the symbol duration, so that we can resolve each multipath independently. If we have $L$ multipaths with delay of the $l$-th path $\tau_{l}$, and the gain of the $l$-th path from the transmit antenna $k$ is $h_{k}^{l}, l=0, . ., L-1$. Then we could write the received signal vector as

$$
\begin{aligned}
{\left[\begin{array}{c}
\mathbf{r}_{0} \\
\mathbf{r}_{1} \\
\vdots \\
\mathbf{r}_{L-1}
\end{array}\right] } & =\left[\begin{array}{c}
\mathbf{H}_{0} \\
\mathbf{H}_{1} \\
\vdots \\
\mathbf{H}_{L-1}
\end{array}\right] \mathbf{x}+\left[\begin{array}{c}
\mathbf{n}_{0} \\
\mathbf{n}_{1} \\
\vdots \\
\mathbf{n}_{L-1}
\end{array}\right] \\
\mathbf{r}_{m p} & =\mathbf{H}_{m p} \mathbf{x}+\mathbf{n}_{m p}
\end{aligned}
$$

where the subscript 'mp' stands for multipath environment. Applying channel matched filtering yields

$$
\mathbf{r}_{m f}=\mathbf{H}_{m p}^{H} \mathbf{r}_{m p}=\Delta_{m p} \mathbf{x}+\mathbf{H}_{m p}^{H} \mathbf{n}_{m p}
$$

Here, it should be noted from this equation that, there is an inherent RAKE receiver which accumulates all the paths after multiplying by their complex conjugates. Similar to the single path case, the quantity $\boldsymbol{\Delta}_{m p}=\mathbf{H}_{m p}^{H} \mathbf{H}_{m p}$ is again a $4 \times 4$ matrix with entries $\gamma_{m p}$ and $\alpha_{m p}$

$$
\Delta_{m p}=\left[\begin{array}{cccc}
\gamma_{m p} & 0 & 0 & \alpha_{m p} \\
0 & \gamma_{m p} & -\alpha_{m p} & 0 \\
0 & -\alpha_{m p} & \gamma_{m p} & 0 \\
\alpha_{m p} & 0 & 0 & \gamma_{m p}
\end{array}\right]
$$

where $\gamma m p=\sum_{l=0}^{L-1} \sum_{k=1}^{4}\left|h_{k}^{l}\right|^{2}$, and $\alpha_{m p}=$ $\sum_{l=0}^{L-1} \operatorname{Re}\left\{h_{1}^{l *} h_{4}^{l}-\quad h_{2}^{l *} h_{3}^{l}\right\}$. Therefore the optimization of multipath channel can be done similar to the single path case as depicted in Section III, where now we have $\kappa=\sum_{l=0}^{L-1} h_{1}^{l *} h_{4}^{l}$ and $\lambda=\sum_{l=0}^{L-1} h_{2}^{l *} h_{3}^{l}$. Hence, as before a feedback for two phasors is adequate to make $\alpha_{m p}$ zero. 
A similar form as in equations (11) - (12) can be obtained when multiple antennas ( $L$ antennas) are used at the receiver, by viewing $h_{k}^{l}$ as the path gain from the $k$-th transmit antenna to the $l$-th receive antenna.

\section{Simplifications of THE FEedBack SCHEME}

Due to practical limitations, the number of feedback bits required from the receiver to the transmitter should be kept as little as possible. One way of achieving this goal is to reduce the number of phase rotations at the transmitter antennas. Another technique is to quantize the feedback phase information. In the sequel, we will address these simplifications, namely reducing the number of feedback bits, while retaining satisfactory forward link performance. Another way of reducing the feedback overhead is to alternatively feedback one phase at a time slot, but this will not be studied in this paper.

\section{A. Single phase feedback}

Instead of rotating the signal from both antennas, only one of them can be rotated. With rotation applied only at the fourth antenna, $\alpha$ can be rewritten as

$$
\begin{aligned}
\alpha & =\operatorname{Re}\left\{h_{1}^{*} h_{4} e^{j \theta}-h_{2}^{*} h_{3}\right\} \\
& =|\kappa| \cos (\theta+\angle \kappa)-\lambda_{r}
\end{aligned}
$$

It can be seen that this is a cosine wave which is a function of $\theta$, scaled by $|\kappa|$, phase shifted by $\angle \kappa$, and biased by $-\lambda_{r}$, where $|\kappa|, \angle \kappa$, and $\lambda$ are as defined in Section III and $\lambda_{r}$ is the real part of $\lambda$. Under the condition $\left|\lambda_{r}\right| \leq|\kappa|, \alpha=0$ has two solutions for $\theta$,

$$
\begin{aligned}
& \theta_{1}=\arccos \left(\frac{\lambda_{r}}{|\kappa|}\right)-\angle \kappa \\
& \theta_{2}=-\arccos \left(\frac{\lambda_{r}}{|\kappa|}\right)-\angle \kappa
\end{aligned}
$$

On the other hand, if $\left|\lambda_{r}\right|>|\kappa|$, there is no solution for $\alpha=0$ and $|\alpha|$ can only be minimized at the following phase value

$$
\theta= \begin{cases}\pi-\angle \kappa, & |\kappa|<-\lambda_{r} \\ -\pi-\angle \kappa, & |\kappa|<\lambda_{r}\end{cases}
$$

with the minimum value

$$
\alpha_{\text {min }}=\left\{\begin{array}{c}
-|\kappa|-\lambda_{r},|\kappa|<-\lambda_{r} \\
|\kappa|-\lambda_{r},|\kappa|<\lambda_{r}
\end{array}\right.
$$

\section{B. Quantization}

The equations (14)-(15) for the single phase and (8)-(9) for the two phase cases, provide optimum phase angles with infinite precision that requires large number of bits to express the floating/fixed point value of the phases. But in practice, only a very low and finite number of bits are allowed for the feedback.

Assume only $\mathrm{K}$ bits are allowed for the feedback. For the single antenna phase adjustment, the discrete estimated feedback information corresponding to the phase $\theta$ will be an element of the set $\left\{\tilde{\theta} \in \Omega=\left\{\frac{2 \pi k}{2^{K}}\right\}, k=0,1, \ldots, 2^{K}-1\right\}$ and is computed as

$$
\tilde{\theta}=\arg \min _{\tilde{\theta} \in \Omega}\left(\left|h_{1}^{*} h_{4} e^{j \theta}-h_{2}^{*} h_{3}\right|\right) .
$$

Similarly, for the dual antenna phase adjustment, the discrete estimated feedback information for the phases $\theta$ and $\phi$ are the elements of the set $\left\{\{\tilde{\theta}, \tilde{\phi}\} \in \Omega=\left\{\frac{2 \pi k}{2^{(K-1)}}\right\}, k=\right.$ $\left.0,1, \ldots, 2^{(K-1)}-1\right\}$ and these are computed as

$$
\{\tilde{\theta}, \tilde{\phi}\}=\arg \min _{\{\tilde{\theta}, \tilde{\phi}\} \in \Omega}\left(\left|h_{1}^{*} h_{4} e^{j \theta}-h_{2}^{*} h_{3} e^{j \phi}\right|\right)
$$

Since the term $\alpha$ may not have been completely cancelled, linear equalization should be employed after matched filtering. We apply a zero-forcing equalizer (but an MMSE or an MLSE equalizer can also be employed)

$$
\begin{aligned}
\mathbf{r}_{z f} & =\boldsymbol{\Delta}^{-1} \mathbf{r}_{m f} \\
& =\left[\begin{array}{llll}
x_{1} & x_{2} & x_{3} & x_{4}
\end{array}\right]^{T}+\boldsymbol{\Delta}^{-1} \tilde{\mathbf{n}}
\end{aligned}
$$

It can be shown that the SNR is

$$
S N R_{z f}=\frac{\gamma^{2}-\alpha^{2}}{\gamma} \frac{\sigma_{x}^{2} / m}{\sigma_{n}^{2}}
$$

where $m$ is the number of transmit antennas (in this study $m=4), \sigma_{x}^{2}$ is the total transmit power of the desired signal, and $\sigma_{n}^{2}$ is the noise power at the receiver. Hence minimization of $\alpha$ maximizes the channel capacity

$$
C=\log _{2}(1+S N R) \text { bits/sec/Hz }
$$

A similar argument can be applied in the multipath and/or multiple receive antenna case, by replacing the terms $\gamma$ and $\alpha$ with $\gamma_{m p}$ and $\alpha_{m p}$ which are given in Section IV.

\section{SIMULATIONS AND RESULTS}

A UMTS FDD model [1] is assumed, with chip rate $3.84 \mathrm{Mcps}$ and carrier frequency $2 \mathrm{GHz}$. A high data rate service with a spreading factor of 8 has been chosen. There are 15 slots in a $10 \mathrm{~ms}$ frame and each slot is 2560 chips long. At the assumed chip rate, there are 320 complex symbols per slot. A modified Jakes' model is used to model the Rayleigh fading channel, [4]. Four uncorrelated fading waveforms are generated to model the channel from each transmit antenna to the receive antenna. Simulations are carried out for various mobile speeds. A feedback delay of two slots is considered so that the phases used to pre-process the transmitted signal during slot $n$ have been computed based upon the channel estimates of slot $n-2$.

Figure 2 depicts the bit error rate (BER) performance of the proposed open and closed loop schemes and compares them with the conventional single transmit single receive antenna scheme, and Alamouti's two transmit one receive antenna STBC scheme [2]. Both single phase and dual phase feedbacks are investigated. Infinite precision is assumed for the feedback phase. In all schemes, for the computation of $E_{b} / N_{o}$, the total transmitted power is considered instead of transmit power per antenna. The mobile speed is $30 \mathrm{~km} / \mathrm{h}$.

All the multi antenna methods outperform the single antenna scheme. The four transmit antenna open loop scheme provides better performance as compared to Alamouti's two antenna scheme. The use of feedback is also justified, by the $2 \mathrm{~dB}$ improvement it affords over the open loop scheme. It can be seen that at a BER of $10^{-3}$, the performance improvement of our proposed feedback technique is approximately 13 


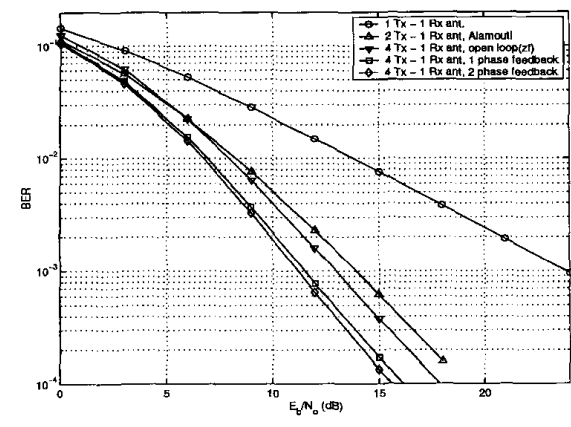

Figure 2: Bit error rate comparison between a conventional one transmit antenna, two transmit antennas(Alamouti) and the proposed four transmit antenna scheme with open loop and with single and dual phase feedback schemes for a UMTS FDD model at mobile speed $30 \mathrm{~km} / \mathrm{h}$.

dB compared to a conventional single antenna scheme and approximately $2.8 \mathrm{~dB}$ compared to Alamouti's two antenna scheme. The two phase feedback scheme performs only 0.4 $\mathrm{dB}$ better than the single phase feedback scheme. Considering the additional overheads due to the feedback information bits, in practice, it is reasonable to opt for the single phase pre-processing scheme.

In Figure 3, the effects of quantization and vehicle speed are investigated for the single phase feedback scheme. The infinite precision and finite precision (two and four bit) phase feedback performances are compared at mobile speeds 3,30 and $50 \mathrm{~km} / \mathrm{h}$. It can be seen that as the speed increases the sensitivity to phase quantization decreases, and two bit feedback achieves adequate performance at all the speeds investigated.

In Figure 4, we investigate the BER performance of the proposed closed loop STBC for a frequency selective fading channel with two equal power paths at $3 \mathrm{~km} / \mathrm{h}$ speed. The performance is compared with one transmit and four receive antenna maximal ratio receive combiner (MMRC). Apart from a $6 \mathrm{~dB}$ gain, the BER curves of the closed loop STBC and the MRRC appear to be identical, confirming the closed loop STBC attains full transmit diversity. The $6 \mathrm{~dB}$ difference in gain is due to the fact that for STBC the total transmit power for both STBC and MRRC schemes is kept the same, i.e. the transmit power per antenna for STBC is a quarter of the total transmit power. In Figure 4, the BER performances of STBC and MRRC for flat fading channel are also given for comparison.

\section{CONCLUSIONS}

We proposed a closed loop STBC technique for four transmit antennas and showed that this scheme attains full transmit diversity with feedbacks for two phasors. It is shown that a significant gain is still achieved when one antenna phase is rotated and the amount of bits allocated for the feedback is limited only to two bits per slot as in the UTRAN FDD system. Moreover, it is shown that the amount of feedback required does not increase with the number of multipath and/or number of receive antennas and two phase feedback is still adequate to attain full diversity.

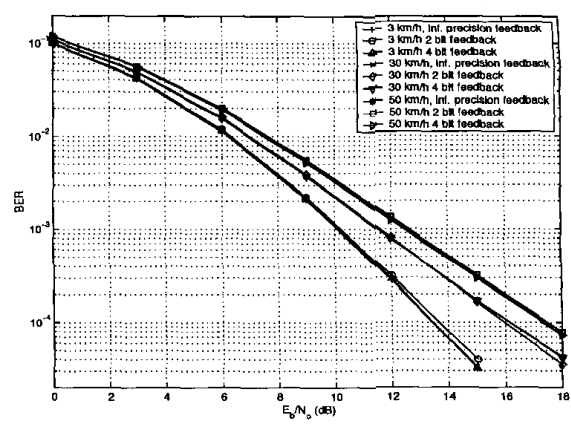

Figure 3: Bit error rate comparison of the proposed four transmit antenna scheme with single antenna phase, infinite and finite ( 2 and 4 bits) precision feedback for a UMTS FDD model at mobile speeds 3,30 and $50 \mathrm{~km} / \mathrm{h}$.

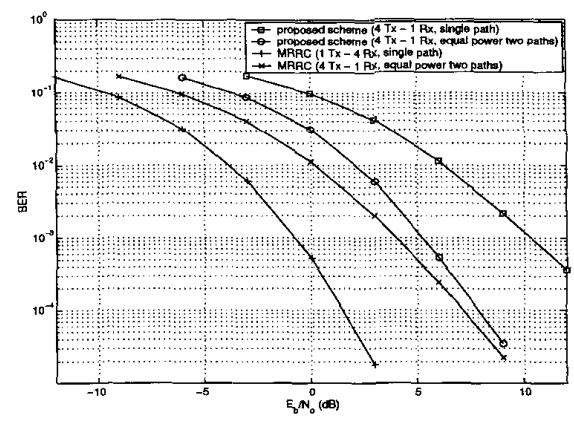

Figure 4: Bit error rate comparison of the proposed four transmit one receive antenna scheme with both single and multipath (two equal power paths) channels having infinite precision feedback and the one transmit four receive antenna maximal ratio combiner over both single and multipath channels for a UMTS FDD model at mobile speed $3 \mathrm{~km} / \mathrm{h}$.

\section{REFERENCES}

[1] 3GPP TS 25.211 V5.0.0, Physical channels and mapping of transport channels onto physical channel (FDD) (Release 5).

[2] S.M. Alamouti, "A simple transmit diversity technique for wireless communications", IEEE J.Select.Areas Commun., vol.16,pp.14511458, Oct. 1998.

[3] H. Jafarkhani, "A quasi-orthogonal space-time block code", IEEE Trans. Commun., vol.49, pp.1-4 , Jan. 2001.

[4] P.Dent, G.E. Bottomley, T.Croft, "Jakes fading model revisited", Electronic Letters, vol.29, pp.1162-1163, 1993.

[5] C.B. Papadias, G.J. Foschini, "A space-time coding approach for systems employing four transmit antennas", IEEE ICASSP 2001, vol.4, pp.2481-2484

[6] N. Sharma, C.B. Papadias, "Improved quasi-orthogonal codes", IEEE WCNC 2002, vol.1, pp. $169-171$

[7] S. Rouquette, S. Merigeault, K.Gosse, "Orthogonal full diversity spacetime block coding based on transmit channel state information for $4 \mathrm{Tx}$ antennas", IEEE ICC 2002, vol.1, pp.558-562.

[8] V. Tarokh, H. Jafarkhani, A.R. Calderbank, "Space-time block codes from orthogonal designs", IEEE Trans. Inform. Theory, vol.45, pp1456-1467, July 1999.

[9] O. Tirkkonen, A. Boariu, A.Hottinen,"Minimal non-orthogonality rate 1 space-time block code for 3+Tx",ISSSTA 2000, pp.429-432, Sept.2000.

[10] C. Toker, S. Lambotharan, J.A. Chambers, "Space-time block coding for four transmit antennas with closed loop feedback", submitted to IEEE Trans. Wireless Commun., May 2002. 